\title{
PROGRAMAS DE EDUCAÇÃO PERMANENTE PARA IDOSOS NO CONTEXTO DA PANDEMIA PELO CORONAVÍRUS: PROPOSTAS E DESAFIOS
}

\author{
Jordelina Schier \\ ORCID: 0000-0001-5117-7234 \\ Juliana Vieira de Araujo Sandri" \\ ORCID: 0000-0002-0606-350X \\ Maria Fernanda Baeta Neves Alonso da Costa' \\ ORCID: 0000-0002-2763-8050 \\ Michele Medeiros' \\ ORCID: 0000-0001-7798-3963
}

'Universidade Federal de Santa Catarina. Florianópolis, Santa Catarina, Brasil.

"Universidade do Vale do Itajaí. Itajaí-SC, Santa Catarina, Brasil.

\section{Autor Correspondente: Jordelina Schier \\ E-mail: jordelina.schier@gmail.com}

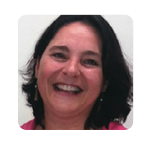

Como citar:

Schier J, Sandri JVA, Costa MFBNA, Medeiros M. Programas de Educação Permanente para Idosos no Contexto da Pandemia pelo Coronavírus: Propostas E Desafios. In: Santana RF (Org.). Enfermagem gerontológica no cuidado do idoso em tempos da COVID 19. Brasilia, DF: Editora ABen; 2021. 171 p. (Serie Enfermagem e Pandemias, 5). https://doi.org/10.51234/aben.21.e05.c19

\section{INTRODUÇÃO}

O mundo, por conta da condição da pandemia pelo novo coronavírus declarada pela Organização Mundial da Saúde (OMS) em 11 de março de 2020, vivencia uma nova e grave problemática de saúde, ainda sem vacina e protocolo terapêutico eficaz, além das repercussões drásticas e incertas no âmbito social, econômico e político da sociedade.

Desde então, as autoridades sanitárias e governamentais adotaram medidas não farmacológicas para reduzir a transmissão do vírus na comunidade, visando retardar a progressão e o impacto da doença nos serviços de saúde, com a implementação de distanciamento social, medidas de etiqueta respiratória, higienização das mãos e superfícies, definição dos casos, isolamento de casos confirmados, uso de máscaras, desenvolvimento de pesquisas sobre o vírus/ formas de combatê-lo e ampla divulgação de informações para orientar as condutas e reduzir o impacto da infecção na sociedade ${ }^{(1)}$.

Com vistas a fornecer direcionamentos práticos para gestores e planejadores de políticas de saúde, desenvolver planos estratégicos de preparação e resposta para o combate à COVID-19, a OMS divulgou o Plano de Contingência Nacional que determina a organização do nível de emergência de saúde pública de importância nacional, sendo que um dos seus pilares é a comunicação sobre os riscos e o engajamento da comunidade. No qual, as universidades possuem papel preponderante para identificar canais preferíveis de comunicação com a comunidade, incluindo aos grupos mais vulneráveis; identificar grupos comunitários e redes locais; e disseminar materiais educativos em locais e canais estratégicos ${ }^{(2)}$.

Em se tratando da vulnerabilidade da população brasileira envelhecida, apesar da pouca efetividade de políticas de promoção do envelhecimento ativo e saudável, os programas de educação permanente com idosos desenvolvidos 
nas universidades, desde a década de 80, promovem a aprendizagem ao longo da vida e a participação cidadã da pessoa idosa, através do conhecimento aplicado e da interação de profissionais qualificados com a pessoa idosa na realidade social e comunitária.

Desse modo, acredita-se que a universidade, por desempenhar papel primordial no ensino, pesquisa e extensão, exercita o seu compromisso social, buscando gerar novos conhecimentos e tecnologias aplicáveis e factíveis no atual contexto de pandemia, especialmente na área da gerontologia.

Considerando as diretrizes locais e da OMS que versam sobre as medidas de quarentena, isolamento e distanciamento sociais, tornou-se imperativo recriar os meios de contato e exercício de educação não formal no ambiente dos programas de educação permanente com idosos.

Desse modo, esse estudo apresenta as experiências desenvolvidas no Núcleo de Estudos da Terceira Idade da Universidade Federal de Santa Catarina (NETI/UFSC) e na Universidade da Vida da Universidade do Vale do Itajaí (UNIVIDA/UNIVALI), neste período de pandemia, e que se configuram como estratégias inovadoras de promoção da saúde em gerontologia.

Este estudo se justifica por se tratar de grupo etário com evidência de gravidade e ampla vulnerabilidade decorrentes do processo de envelhecimento e ocorrência de comorbidade ${ }^{(1)}$ diante da contaminação pelo novo Coronavírus. A relevância, se deve pela abrangência em buscar estratégias de inovação social e tecnológica em educação permanente, com vistas a manutenção do vínculo social, suporte em saúde, ações socioeducativas necessárias para a prevenção de danos decorrentes do distanciamento social, além da otimização do estreitamento das relações sociais e familiares, principalmente pós-pandemia ${ }^{(3)}$.

\section{OBJETIVOS}

Geral: Apresentar as estratégias para promoção da saúde desenvolvidas em programas de educação permanente com idosos, no âmbito universitário, em contexto de pandemia pelo coronavírus.

Específicos:

- Descrever as atividades de educação permanente com vistas à promoção da saúde;

- Discutir as inovações tecnológicas nos programas de educação permanente;

- Refletir sobre a atuação da (o) enfermeira (o) em equipe multiprofissional nos programas de educação permanente.

\section{MÉTODO}

O Relato de Experiência é uma ferramenta da pesquisa descritiva que aborda e descreve uma situação ou uma prática que devem ser relacionadas com evidências científicas. Optou-se pela pesquisa descritiva, pois esta permite realizar uma análise minuciosa dos fatos a fim de descrevê-los ${ }^{(4)}$ e abordagem qualitativa para elucidar as estratégias de promoção da saúde desenvolvidas nos programas de educação permanente com idosos do NETI/UFSC e UNIVIDA/UNIVALI, em contexto de pandemia pelo coronavírus, atualmente realizados a distância.

\section{O Cenário dos Programas de Educação Permanente com Idosos nas Universidades Brasileiras}

Os programas de educação permanente com idosos nas universidades foram criados a partir da experiência da Universidade de Ciências Sociais de Toulouse, na França dos anos 70, e alcançaram o mundo sob a denominação de universidade aberta da terceira idade (UNATI). Desde então, diferentes modelos e formas de organização foram implementados nas instituições de ensino superior respeitando a realidade local, o público, os objetivos e metodologias, e os recursos disponíveis no seu contexto ${ }^{(5-6)}$. Hoje, as UNATI representam uma janela de oportunidades para aprender, ensinar, pesquisar e conviver, sendo um local propício para 
gerar novos conhecimentos e desenvolver um novo paradigma sobre a velhice, onde o desenvolvimento está presente em toda existência humana ${ }^{(7)}$.

No Brasil, o programa pioneiro foi o NETI/UFSC, criado em 1982 numa época em que as projeções de envelhecimento populacional ainda não haviam se concretizado e, por isso, participou de lutas sociais em prol dos direitos da pessoa idosa e de afirmação institucional por ocupação de espaço e conquista de recursos para, finalmente, firmar-se como um modelo de intervenção gerontológica com projeção nacional.

O NETI é um programa vinculado à Pró-Reitoria de Extensão da UFSC, que desenvolve atividades de ensino, pesquisa e extensão a pessoas com 50 anos e mais. $O$ ensino ocorre na modalidade não formal e, semestralmente, oferta atividades socioeducativas, cujo acesso se dá por meio de Edital publicado no site oficial do programa. A equipe de trabalho é composta por coordenador do Núcleo, assistente social, técnico em assuntos educacionais, enfermeiro, chefe de divisão de apoio administrativo e assistente em administração, em conjunto, com docentes e técnicos dos Centros de Ensino da Universidade. Os projetos desenvolvidos e todas as ações socioeducativas seguem o referencial do campo da gerontologia e da concepção de educação ampla e participativa, segundo Paulo Freire e Relatório Delors da UNESCO.

A missão do NETI é "Redescobrir, recriar de forma integrada, sistematizar e socializar o conhecimento de gerontologia, desenvolvendo atividades de promover as pessoas da terceira idade no meio acadêmico e comunitário, como sujeitos em transformação e transformadores" traz o sujeito que transforma ao mesmo tempo em que é transformado, a ação protagonista da pessoa, desenvolvida por meio da educação permanente ${ }^{(8)}$.

Experiências e projetos de ensino, pesquisa e extensão desenvolvidos pelos docentes e acadêmicos da UFSC resultaram em iniciativas exitosas, de organização de grupos e associações, protagonizadas pelos idosos, que continuam multiplicando os saberes construídos na universidade e aplicados na comunidade, tais como: Monitores da Ação Gerontológica, Intercâmbio Comunitário em Gerontologia, Centro de Estudantes do NETI, Blog IntegraNETI, Grupo A Hora da História; Grupo de Canto Vozes da Ilha; Grupo de Dança Renascer, Grupo de Encontros Culturais, Grupo de Apoio às Pessoas com Parkinson e seus Familiares, dentre outros.

O programa UNIVIDA também foi se construindo historicamente, sendo originário de outras atividades de educação permanente designada para as pessoas na maturidade. Em 1996, tinha-se o Curso AtividadeFaculdade da Vida com o objetivo de promover a qualidade de vida e atualização de conhecimentos para os funcionários da instituição de ensino superior. Em 1998, o referido curso foi remodelado e ampliado para fora dos muros universitários, denominado de PIMI- Programa Institucional da Maior Idade, com módulos temáticos. Em 2000, a UNIVALI estava em processo de institucionalização dos projetos de extensão, os quais foram reordenados dentro do ciclo vital, muitos foram extintos e outros reorganizados. O PIMI foi incorporado para um projeto maior, o Curso Superior de Extensão - Universidade da Vida (UNIVIDA), do Centro de Ciências da Saúde, e agora, a Escola de Ciências da Saúde ${ }^{(9)}$.

Atualmente, o UNIVIDA, está incorporado aos Programas Institucionais de Extensão e vinculado a Pró-Reitoria de Extensão e Cultura, é coordenado pelo Curso de Graduação de Enfermagem. Para melhor atender a demanda dos alunos idosos, o programa se apresenta em duas vertentes: UNIVIDA I e II. Sendo que o primeiro tem duração de dois anos, entrada anual, matriz curricular definida e clientela composta por pessoas com mais de 40 anos, com o objetivo de desenvolver atividades que promovam o envelhecimento saudável, a qualidade de vida e a atuação voluntária. As disciplinas abordam temas das áreas das ciências da saúde, ciências sociais e humanas. Os projetos de atuação voluntária oriundos do UNIVIDA I tiveram várias ações exitosas e duradouras, a exemplo do Grupo de Apoio a Pessoas com Alzheimer (GEAz), Grupo de Canto UNIVIDA, Contação de histórias e outros. Já o UNIVIDA II tem o propósito de dar continuidade aos conteúdos desenvolvidos na proposta do UNIVIDA I. Destaca-se que ambos são campos de atividades de ensino e pesquisa em que ocorrem intercâmbios de vários Cursos de Graduação e Pós-Graduação da Escola de Ciências da Saúde e da Escola de Ciências Jurídicas. Vários projetos de iniciação científica e de dissertação do Mestrado Profissional em Saúde e Gestão do Trabalho desenvolveram pesquisas sobre tecnologias leves junto a este público, assim 
como realizaram atividades práticas de ensino, via projeto de atuação, vinculada ao tema da dissertação. Há também acadêmicos extensionistas que trazem suas experiências de extensão, a exemplo das atividades realizadas no Grupo de Afásicos, do Escritório modelo do Curso de Direito, entre outros.

De todo modo, os programas de educação permanente para os idosos no âmbito universitário realizam ações promotoras do envelhecimento ativo e saudável, auxiliando a pessoa idosa a manter sua cidadania e autonomia, além dos efeitos que repercutem na revisão dos mitos e preconceitos da sociedade frente ao envelhecer e velhice. Desse modo, configuram-se em importantes equipamentos educativos e de integração social, sem caráter assistencialista, e que geram oportunidades relacionadas à quebra de estereótipos, visto que a universidade estimula a reflexão e dissemina conhecimentos. Também são ricos campos de pesquisas em diversas áreas, que podem ser desenvolvidas para e com os idosos, e de modo intergeracional, mobilizando seus conhecimentos, criatividade, inteligência e memória ${ }^{(10)}$.

Diante da situação pandêmica pelo coronavírus, bem como das determinações do Ministério da Saúde que versam sobre o estado de emergência sanitária e as medidas de enfrentamento da transmissão comunitária ${ }^{(1,11-12)}$, as universidades em questão, UFSC e UNIVALI, deliberaram oficialmente a suspensão das atividades presenciais de ensino. Igualmente, as atividades de extensão nos programas de educação permanente com idosos, até então realizadas exclusivamente de modo presencial, foram suspensas e as equipes multiprofissionais, mantendo o compromisso social, o trabalho educativo e o vínculo afetivo com o público da UNATI buscaram meios tecnológicos para o atendimento da pessoa idosa, com a manutenção do sentimento de pertencimento ao programa e com ações de suporte de promoção da saúde em situação de isolamento/distanciamento sociais.

\section{Promoção da Saúde no Contexto de Pandemia pelo Coronavírus: novas práticas de educação per- manente com idosos}

A promoção da saúde é um dos pilares, que juntamente com a aprendizagem ao longo da vida, participação e segurança/proteção, favorecem o envelhecimento ativo. As ações socioeducativas, com foco na promoção da saúde, disponibilizam conhecimentos necessários para que a pessoa possa cuidar melhor da própria saúde e seja agente multiplicador de tais conhecimentos na sua família e comunidade ${ }^{(13)}$. Os profissionais qualificados contribuem para o desenvolvimento de uma cultura de cuidado frente ao fenômeno do envelhecimento, em ambiente interativo de educação em saúde, de modo compartilhado e com responsabilidades coletivas, no contexto dos programas de educação permanente com idosos. Especialmente na atual situação de crise sanitária e incertezas de toda ordem.

O Programa Quarentena no NETI, idealizado pela equipe multiprofissional do Núcleo, teve seu início em 30/03/2020 com o objetivo de manter o vínculo com os estudantes do NETI, durante o período da quarentena, com vistas à promoção da saúde. O Programa é desenvolvido através da veiculação de materiais sobre o coronavírus e outros temas, todas as segundas e quintas feiras, nos grupos de WhatsApp dos 446 estudantes idosos inscritos nas atividades socioeducativas ofertadas pelo NETI. Os conteúdos publicados são pesquisados pela equipe, composta pela Coordenação; Assistente Social, Enfermeira, Técnico em Assuntos Educacionais e dois servidores administrativos. Os materiais possuem cunho educativo voltado para saúde, bem-estar e a promoção da saúde dos estudantes idosos, no período da pandemia.

A preparação das atividades enviadas aos estudantes idosos ocorre da seguinte forma: 1- a equipe sugere os materiais no seu grupo do WhatsApp, no dia anterior a postagem; 2- a equipe discute e define os materiais a serem postados; 3 - um membro da equipe elabora o texto da postagem e anexa os materiais selecionados; 4- o material completo de postagem é revisado; 5 - após confirmação da equipe, a postagem é repassada para um membro da equipe responsável pela veiculação nos grupos de WhatsApp dos estudantes idosos; 6- no dia seguinte, o texto com os materiais são postados. O Programa Quarentena no NETI, até a data de 07/07/20, apresentou 29 episódios, totalizando a postagem de 80 materiais sobre a prevenção, transmissão e cuidados com a COVID-19, além de outros conteúdos relativos a saúde mental, cognição e memória, de cunho artístico, 
educativo, entretenimento, autoajuda, vídeos diversos, arte e cultura através de visitas virtuais, cartilhas, livros, entrevistas, lives e e-books, dentre outros. O NETI recebeu feedback dos alunos idosos satisfeitos com o conteúdo e formato do Programa, certamente, há a previsão de aplicar avaliação sistemática desse recurso que, decorrente de um momento emergencial, vem sendo testado e repensado dia a dia, mantendo a missão norteadora do NETI, bem como a ética e compaixão que rege o cuidado com pessoas.

O programa UNIVIDA, nas suas duas vertentes, manteve os cursos com aulas em ambiente virtual de aprendizagem, organizando e desenvolvendo suas atividades por meio de videoaulas no sistema Blackboard Collaborate por lives e redes sociais. A manutenção do contato com os alunos foi considerada primordial, mesmo de modo virtual, visto que muitos vivem só e, portanto, estão vulneráveis a desenvolverem ou intensificar os problemas psicológicos, sociais e físicos já existentes.

A tecnologia de ensino remoto institucionalizado pela UNIVALI, com o sistema Blackboard Collaborate, corresponde a uma plataforma de tecnologia de educação, onde o professor pode gerar um link de acesso público que comporta até 250 participantes. Tal plataforma permite a interação sincrônica e assincrônica com troca de materiais, conversas e e-mails, todas as atividades são gravadas e podem ser acessadas posteriormente.

Assim que ficou decidido que todas as aulas passariam a ser remotas, criou-se um grupo no WhatsApp com todos os alunos do UNIVIDA I e II (42 alunos) para que houvesse um único meio de comunicação para informar sobre a dinâmica das atividades. O WhatsApp foi a tecnologia escolhida devido a familiaridade de uso junto aos alunos idosos, por ser um aplicativo de multiplataformas e, portanto, de fácil acesso. A coordenadora do UNIVIDA I e II, junto com a bolsista, é responsável por manter os alunos atualizados, semanalmente, sobre os temas e atividades. O planejamento e o cronograma foram socializados no WhatsApp e nas demais mídias sociais (Facebook e Instagram).

As atividades elaboradas para esse tempo remoto foram construídas pelos professores da área da saúde, das humanas e jurídica atuantes na UNIVIDA e disponibilizadas nos formatos de vídeos, lives, oficinas, roda de conversa on line, entre outras estratégias de ensino. As temáticas trabalhadas corresponderam: 1) Bons hábitos e Vida Saudável com o foco na prevenção de contaminação da Helicobacter pylori. 2) Técnicas de gastronomia e armazenamento de alimentos. 3) Envelhecimento da Voz, tratando sobre técnicas de cuidado com a voz e avaliação vocal. 4) Hidratação da pele a partir dos alimentos (vitamina D) e a necessidade do sol. 5) Imunização no Idoso, abordando sua importância e sanando dúvidas. 6) Resiliência e a interação social em época de isolamento social.7) Direito do Empregado Doméstico. 8) Exercício Físico. 9) Musicoterapia para a saúde mental. 10) Qualidade de vida no idoso, como fechamento do semestre avaliando o quanto as atividades remotas contribuíram para manter o isolamento social de modo mais saudável.

Considerando que, a maioria das pessoas idosas que frequenta uma UNATI apresenta, ou está desenvolvendo, habilidade digital para utilizar o correio eletrônico, usar os mecanismos de busca de informações na Internet, bem como as redes sociais (Facebook, Twitter, Instagram, WhatsApp), a utilização de tais recursos tecnológicos foi a opção possível e mais adequada para manter o trabalho nas UNATI.

Os avanços das novas tecnologias da informação e comunicação, assim como a popularização do uso de redes sociais, são chaves no cotidiano das pessoas. Embora a inclusão digital de pessoas idosas seja um desafio para a educação permanente, a internet e as redes sociais surgem como um grande suporte para um envelhecimento ativo e deve ser considerado no desenvolvimento das políticas da sociedade atual. A interação social que os idosos alcançam quando participam de redes sociais os mantêm comunicados, ativos e constantemente aprendendo a resolver desafios ${ }^{(14)}$.

\section{Atuação da (o) enfermeira (o) em equipe multiprofissional nos programas de educação permanente em gerontologia}

A Gerontologia é a ciência que dialoga e confronta os saberes de diferentes áreas, tecendo uma rede de conhecimentos intercomplementares. Na prática, a atenção ao idoso envolve diversos profissionais que 
compõem a equipe multiprofissional, na qual cada membro deve assegurar a especificidade teórica e prática do seu campo de saber, porém, mantendo atuação articulada ${ }^{(5)}$.

Nesse sentido, a (o) enfermeira (o) utiliza os conhecimentos científicos da gerontologia, a concepção de cuidado integrado com a multidimensionalidade do viver e envelhecer e a disponibilidade de recursos para a conformação de serviços que melhor atendam às demandas do ser idoso, especialmente, àquelas relativas à promoção da saúde, longevidade, autonomia e independência. Nesses serviços, a atuação da (o) enfermeira (o) requer uma atitude cooperativa com os demais membros da equipe multiprofissional, de modo interdependente, assumindo caráter de entreajuda e de crescimento mútuo pessoal e profissional. De modo a desenvolver ações com vistas a valorização das capacidades e potencialidades, minimização das perdas decorrentes do envelhecimento e a manutenção da melhor qualidade de vida possível do público idoso ${ }^{(15)}$.

No que se refere aos programas de educação permanente em gerontologia, a atuação pioneira e inovadora da (o) enfermeira (o), juntamente com uma assistente social, viabilizou a criação da primeira universidade aberta da terceira idade (UnATI) no Brasil, reconhecida nacionalmente como um modelo de intervenção gerontológica que realiza educação permanente para o envelhecimento e promove a inclusão da pessoa idosa no meio universitário, o NETI/UFSC.

Desde então, o NETI conta com a presença da (o) enfermeira (o) em seu quadro de pessoal, atuando de forma interdisciplinar, cuja principal função tem sido a de coordenar e/ou fornecer suporte técnico em saúde em todas as atividades neste desenvolvidas. Dentre as funções, além da participação na proposição e condução de atividades socioeducativas oferecidas aos idosos e adultos maduros, cita-se a coordenação geral do Núcleo. Tal coordenação implica na articulação de diferentes áreas do conhecimento aos interesses do NETI e do público idoso, seja desenvolvendo pesquisas gerontológica; na representação junto à órgãos municipais, estaduais e federais afins a questão do envelhecimento; no ensino, por meio da participação em atividades curriculares da universidade e de formação lato sensu em gerontologia, supervisão de trabalhos acadêmicos, estágios e bolsas; sempre tendo como enfoque o desenvolvimento da gerontologia e do cuidado em saúde e de enfermagem à pessoa idosa no âmbito de uma UNATI.

De modo semelhante, com caráter multiprofissional, a UNIVIDA/UNIVALI foi idealizada reunindo a psicologia, enfermagem e biologia. Tendo sido iniciada como um projeto de extensão construído com a atuação de diversas áreas do conhecimento e entre as Escolas da UNIVALI. Assim, o projeto exitoso desde 1996, concretizou-se com a atuação multidisciplinar voltada a um único objetivo, o de desenvolver ações de educação permanente focada na promoção da saúde na perspectiva de um envelhecer saudável e na preparação ao voluntariado, a fim de manter a pessoa idosa ativa na sociedade, o maior tempo possível, e empoderada para gerir a própria vida.

Sendo assim, as UNATI perceberam a necessidade de maior engajamento da equipe de trabalho no uso das tecnologias de mídias digitais, incluindo o debate de temas e condutas em reuniões virtuais, além da busca por aperfeiçoamento por meio digital. E, desse modo, estratégias que envolvem o uso de tecnologias intuindo manter o vínculo com seus alunos idosos estão sendo implementadas. Essa preocupação com a manutenção do vínculo, além da produção de trabalho, perpassa por todos os membros da equipe e os professores/facilitadores.

Desse modo, a (o) enfermeira (o), na coordenação dos programas, tem importante papel na articulação dos membros da equipe de trabalho, dos professores/facilitadores e demais participantes, visando o planejamento, manutenção e avaliação dos projetos e atividades em andamento, agora direcionados para a modalidade de teletrabalho. A equipe multiprofissional vem desenvolvendo estratégias de ação e buscando por insumos de qualidade, com diversificados temas e enfoques, que despertem o interesse do público idoso e que agreguem informações acessíveis e contribuintes para o bem estar das pessoas, no contexto de isolamento/distanciamento sociais em tempos de pandemia. 


\section{CONSIDERAÇÕES FINAIS}

As universidades da terceira idade representam importante equipamento social e educativo para o envelhecimento ativo e saudável. Com o advento da COVID-19 e sua rápida transmissão em escala mundial, tais instituições, sem uma programação prévia, tiveram que reorganizar suas dinâmicas de trabalho e de interação com os alunos idosos.

A reorganização dos trabalhos tem implicado no enfrentamento de desafios de toda ordem, sejam aqueles estruturais físicos, materiais e equipamentos, acesso à internet, tecnologias educativas e de saúde, até aqueles que envolvem a capacidade de organização e desenvolvimento dos profissionais, e da equipe como um todo, em busca de subsídios para a criação e/ou implemento de ações que configurem uma resposta da instituição ao seu público.

Algumas universidades da terceira idade vêm implementando diferentes propostas com vistas a vencer esses desafios, mobilizando a equipe interdisciplinar para adequar o uso da tecnologia de mídias digitais para a concretização de suas ações socioeducacionais e interativas. Os Programas NETI/UFSC e UNIVIDA/ UNIVALI vêm se reinventando em tempos de pandemia, experienciando o trabalho remoto da equipe multiprofissional e desenvolvendo estratégias criativas de ensino das temáticas que possam maximizar as atitudes de promoção da saúde de seus alunos idosos, e minimizar os efeitos do distanciamento social, enquanto a relação presencial não for possível.

A atuação interdisciplinar da (o) enfermeira (o) na equipe cumpre a sua função específica de cuidado com a pessoa idosa, sem perder de vista a interdependência com os demais profissionais, visando à promoção de um envelhecimento ativo e saudável, com foco na educação para o cuidado com a vida e a saúde.

\section{AGRADECIMENTO}

Ao Departamento Científico de Enfermagem Gerontológica da ABEn Nacional

\section{REFERÊNCIAS}

1. Ministério da Saúde (BR). Secretaria da Vigilância em Saúde. Boletim Epidemiológico no 05 [Internet]. 2020 [cited 2020 Jun 23]. Available from: https://portalarquivos.saude.gov.br/images/pdf/2020/março/24/03--ERRATA---BoletimEpidemiológico-05.pdf

2. Albuquerque NLS. Planejamento operacional durante a pandemia de Covid-19: comparação entre recomendações da Organização Mundial da Saúde e o Plano de Contingência Nacional. Cogitare Enferm. 2020;25. https://doi.org/10.5380/ ce.v25i0.72659

3. Hammerschmidt KSA, Bonatelli LCS, Carvalho AB. Caminho da esperança nas relações envolvendo os idosos: olhar da complexidade sob a pandemia do COVID-19.. Texto Contexto Enferm. 2020;29:e20200132. https://doi. org/10.1590/1980-265x-tce-2020-0132

4. Gerhardt TE, Silveira DT. Métodos de pesquisa. Universidade Aberta do Brasil - UAB/UFRGS e pelo Curso de Graduação Tecnológica - Planejamento e Gestão para o Desenvolvimento Rural da SEAD/UFRGS. Porto Alegre: Editora da UFRGS; 2009.

5. Doll J. Multidimensionalidade do Envelhecimento e Interdisciplinaridade. In: Freitas EV, PY L. Tratado de Geriatria e Gerontologia 4. ed. Rio de Janeiro: Guanabara Koogan; 2016. p 107-113.

6. Cachioni M, Ordonez TN. Universidade da Terceira Idade. In: Freitas EV, PY L. Tratado de Geriatria e Gerontologia 4.ed. Rio de Janeiro: Guanabara Koogan; 2016. p 1604-1612.

7. Cachioni M. Universidade da Terceira Idade: história e pesquisa. Kairós Gerontol. 2012;15:1-8. https://doi. org/10.23925/2176-901X.2012v15iEspecial14p1-8

8. Núcleo de Estudos da Terceira Idade (NETI). Universidade Federal de Santa Catarina. Apresentação [Internet]. 2020[cited 2020 Jun 23]. Available from: https://neti.ufsc.br/apresentacao/ 
9. Ploner KS. Trajetória da extensão no Curso de Psicologia da UNIVALI: alguns momentos relevantes. In: Sardá, Jamir; Delvani, Josiane da Silva (Org). Curso de Psicologia: 30 anos de atuação na profissão. Universidade do Vale do Itajaí: Itajaí, 2017. p 59-90.

10. Silva FM, Souza I, Schier J. O Empoderamento da Pessoa Idosa para o exercício da Cidadania: educação permanente por meio da extensão universitária [Internet]. In: XVI Colóquio Internacional Sobre Gestión Universitária, 2016, Arequipa, Peru. Anais. Florianópolis: UFSC, 2016[cited 2020 Jun 23]. Available from: https://repositorio.ufsc.br/xmlui/handle/123456789/1 71012?show=full. Acesso em: 06 jul. 2020

11. Presidência da República (BR). Lei n 13.979 em 06 de fevereiro de 2020. Dispõe sobre as medidas para enfrentamento da emergência de saúde pública de importância internacional decorrente do coronavírus responsável pelo surto de 2019 [Internet]. Diário Oficial da União. 07 de Fevereiro de 2020 [cited 2020 Jun 23]. Available from: http://www.in.gov.br/en/ web/dou/-/lei-n-13.979-de-6-de-fevereiro-de-2020-242078735

12. Ministério da Educação (BR). Portaria Normativa $N^{\circ} 354 / 2020 / G R$ de 18 de março de 2020 . Estabelece medidas complementares às portarias normativas n 352/2020/GR e no 353/2020/GR, de 16 de março de 2020, e define prazos sobre o funcionamento das atividades administrativas e acadêmicas na UFSC [Internet]. Universidade Federal de Santa Catarina: Gabinete da Reitoria. Florianópolis, 2020 [cited 2020 Jun 23]. Available from: https://noticias.paginas.ufsc.br/ files/2020/03/portaria-corona-354-2020.pdf

13. Centro Internacional de Longevidade Brasil. Envelhecimento ativo: um marco político em resposta à revolução da longevidade [Internet]. 2015[cited 2020 Jun 23]. 119 Págs. Available from: https://prceu.usp.br/usp60/wp-content/ uploads/2017/07/Envelhecimento-Ativo-Um-Marco-Politico-ILC-Brasil_web.pdf

14. González AJG, Gómez-Millán MRB, Rubio LR. Competencias comunicativas mediadas em estudiantes universitarios mayores: alfabetización tecnológica como experiencia innovadora. Rev Latinoam Tecnol Educ [Internet]. 2017 [cited 2020 Jun 23];16(1). https://doi.org/10.17398/1695-288X.16.1.67

15. Gonçalves LHT, Alvarez AM, Santos SMA. Cuidados de Enfermagem Gerontológica: conceito e prática. In: Freitas EV, PY L. Tratado de Geriatria e Gerontologia 4.ed. Rio de Janeiro: Guanabara Koogan; 2016. p 1247-54.

16. Ministério da Saúde (BR). Coronavírus: 826.866 pessoas estão curadas em todo o Brasil [Internet]. 2020 [cited 2020 Jun 23]. Available from: https://www.saude.gov.br/noticias/agencia-saude/47150-coronavirus-826-866-pessoas-estao-curadas-em-todo-o-brasil 